Classification

Physics Abstracts

$62.20-46.30$

\title{
Une approche micromécanique du comportement des polycristaux
}

\author{
G. Cailletaud
}

Centre des Matériaux, Ecole des Mines de Paris, B.P. 87, 91003 Evry Cedex, France

(Reçu le 26 mai 1987, accepté le 31 aout 1987)

\begin{abstract}
Résumé - Entre les approches phénoménologiques classiques (macro-macro) et les théories de lignes de glissement (micro-macro), on montre ici la possibilité d'emprunter une troisième voie pour la description du comportement inélastique multiaxial des polycristaux : dans les modèles développés (macro-micro) on introduit des lois de comportement macroscopiques au nıveau microscopique, en utilisant un formalisme général de type autocohérent. Les possibilités de ces modèles sont illustrées par une identification sur des chargements uniaxiaux et multiaxiaux, proportionnels ou non, sur un acier inoxydable austénitique 17-12 $\mathrm{SPH}$ (type $316 \mathrm{~L})$. On retrouve un bon agrément général avec l'ensemble des données.
\end{abstract}

\begin{abstract}
The classical theories describing the inelastic multiaxial behaviour of polycrystals are the macro-macro phenomenological approach (macroscopic hardening laws to obtain the macroscopic behaviour) and the micro-macro slip theories (microscopic hardening laws to obtain the macroscopic behaviour). In this paper, a third approach is shown, using macroscopic hardening laws in a self consistent type framework (macro-micro model). The prediction capabilities of this last model are demonstrated through numerical simulations of onedimensional or twodimensional (proportional or nonproportional) loading paths. The results are compared with experimental data on $316 \mathrm{~L}$ stainless steel at room temperature, and the general agreement is good.
\end{abstract}

\section{Introduction}

Deux voies peuvent être suivies pour décrire le comportement inélastique des métaux.

(1) La première utilise des lois de comportement phénoménologiques : de gros progrès ont été effectués dans cette voie au cours des dix dernières années, le succès de ces théories étant principalement lié à la simplicité de leur installation dans des codes éléments finis, et à leur structure modulaire. Leur point de départ commun tient en l'utilisation d'un potentiel plastique et à la définition de l'incrément de déformation plastique à l'aide d'une règle de normalité. L'écrouissage produit une translation, plus une déformation isotrope ou anisotrope de la surface de charge. Un tel type de modèle permet de décrire l'effet Bauschinger, le durcissement cyclique, l'effet de mémoire du chemin de chargement et même le durcissement supplémentaire lié à la non proportionnalité du chargement, ou encore les changements de phase du métal si des variables additionnelles appropriées sont introduites. En viscoplasticité, on pourra représenter le vieillissement, la restauration, et n'importe quel type d'évolution des propriétés mécaniques avec le temps ou la température. Ce type d'approche pose néanmoins un problème de fond lorsqu'il s'agit d'effectuer des prévisions hors du domaine d'identification. La détermination du modèle dans un domaine donné revient en effet à représenter l'image macroscopique d'un ou plusieurs phénomènes microscopiques, si bien que la forme obtenue ne reste valable que si ces mécanismes microscopiques restent prépondérants. Les possibilités de prévision des modèles sont donc étroitement liées à une connaissance approfondie de l'état microstructural.

(2) Dans un second type d'approche, la déformation plastique macroscopique est obtenue comme la sommation de déformations microscopiques sur les systèmes de glissement. Ces théories de lignes de glissement sont basées sur la loi de Schmid, et, dans ce cas, l'écrouissage est décrit par l'évolution de la contrainte de cisaillement critique sur chaque système. Ces modèles ne sont évidemment pas aussisimples àmanipuler que les modèles macroscopiques, mais ils contiennent naturellement un certain nombre d'effets bien connus en plasticité, comme la distorsion de la surface de charge ou l'effet d'écrouissage croisé. Ils sont essentiellement utilisés pour simuler des chargements monotones ou pour décrire des textures, en présence de déformations importantes. Dans ce cas, la relation contrainte-déformation, écrite au niveau microscopique, est largement dépendante de la microstructure. On peut alors parler d'approche micro- macro dans la mesure où l'expression de l'écrouissage au niveau microscopique débouche sur une description du comportement élastique macroscopique. La struc- 
ture physique du modèle (grains, systèmes de glissement) augmente bien sûr la sûreté des prévisions. A l'inverse, on rencontre les limites de l'approche à chaque fois que l'état microscopique est si complexe qu'on ne peut pas faire ressortir de mécanisme microscopique prépondérant, ce qui rend hasardeux le passage micro-macro.

On souhaite donc dans ce texte montrer la possibilité d'emprunter une troisième voie, dans laquelle les lois de comportements macroscopiques classiques sont utilisées sur des variables microscopiques. La structure polycristalline est prise en compte par une représentation de type autocohérent. Les lois d'écrouissage sont écrites en viscoplasticité au niveau du système de glissement : on définit pour chaque système une variable d'écrouissage isotrope et une variable cinématique. On cherche ainsi à réunir les avantages des deux approches précédentes : l'écriture simplifiée de l'écrouissage (sans référence directe à l'état de la microstructure) permet un traitement simplifié du modèle tandis que la forme des interactions entre systèmes de glissement est préservée (matrice d'écrouissage essentiellement).

Les possibilités du modèle sont illustrées par une identification sur des chargements uniaxiaux et multiaxiaux, proportionnels ou non, sur un acier inoxydable austénitique 17-12 $\mathrm{SPH}$. On obtient une bonne description des essais de traction ou de torsion en chargement simple, ainsi que le durcissement supplémentaire qui est lié aux chargements non proportionnels. On n'utilise pour cela que huit coefficients dépendants du matériau (plus les coefficients de la matrice d'écrouissage). On discute alors les résultats que l'on peut attendre d'un tel type de modèle et on montre comment ils peuvent servir de guide pour les développements futurs des modèles macroscopiques.

\section{L'écrouissage dans les modèles macroscopiques}

On donne ici une brève description de l'écriture de l'écrouissage dans les modèles macroscopiques viscoplastiques, en montrant essentiellement la partie de ces modeles qui sera reprise au moment de l'écriture de l'écrouissage à l'échelle microscopique. On trouve de très nombreuses expressions différentes, dans une littérature maintenant abondante, qui porte tant sur le développement des modèles $|1|$ que sur leur installation dans les codes de calcul $|2|$. Dans les métaux, la plupart des auteurs introduisent un domaine élastique initial défini à l'aide du critère de Misès, qui peut ensuite être translaté et déformé par l'écrouissage. L'expression la plus simple est donc obtenue en utilisant une variable scalaire $R$ pour définir le rayon du domaine élastique et une variable tensorielle $\widetilde{\mathrm{X}}$ pour positionner son centre. Le domaine élastique est alors défini par :

$$
f=J(\tilde{\Sigma}-\tilde{X})-R-k<0
$$

On utilise ici les notations de $|3|$, le terme $k$ représentant la limite élastique initiale du matériau en traction simple, les variables $X$ et $R$ caractérisant respectivement l'écrouissage cinématique et l'écrouissage isotrope macroscopiques. Dans ce type d'écriture, le domaine reste une sphère dans l'espace des contraintes, et est donc isotrope autour de son centre. Dans le cas du modèle qui sera choisi comme référence dans la suite de l'article $|4|$, on utilise comme point de départ pour la part isotrope une expression de $R$ qui se sature avec la déformation viscoplastique cumulée $\mathbf{v}$ :

$$
R=Q(1-\exp (-b v))
$$

où $Q$ et $b$ sont des coefficients caractéristiques du matériau. On note que la saturation de la fonction est impérative si $l$ 'on veut appliquer le modèle en chargement cyclique, faute de quoi le domaine élastique augmenterait cycle après cycle jusqu'à ce que l'écoulement viscoplastique disparaisse. L'évolution la plus simple que l'on puisse considérer pour l'écrouissage cinématique est donnée par la règle d'écrouissage cinématique linéaire de Prager, mais on adjoint habituellement un terme de rappel $|5|$ qui permet de limiter l'écrouissage, sous la forme :

$$
\dot{\tilde{x}}=C \dot{\widetilde{E}}_{v}-D(v) \cdot \tilde{x} \cdot \dot{v}
$$

L'adoption d'une telle formulation conduit en fait à introduire un domaine admissible pour la variation de $\widehat{\mathrm{X}}$ dans l'espace des contraintes, de rayon $C / D$, si l'on appelle $D$ la valeur limite de la fonction $D(v)$ lorsque $v$ augmente indéfiniment. On construit ainsi un cas particulier de modèles multisurfaces $|6|$ avec comme unique surface active, le domaine élastique. On note que de nombreuses variantes peuvent être proposées à ce niveau $|7|$. C'est ainsi par exemple qu'une modification de la direction du terme de rappel peut conduire à des comportements identiques en chargement simple, mais radicalement différents sous sollicitations multiaxiales non proportionnelles $|8|$ :

$$
\dot{\tilde{X}}=(C-D \cdot \tilde{\mathrm{X}}: \tilde{n}) \dot{\tilde{E}}_{\mathrm{v}}
$$

où $\tilde{n}$ est le vecteur normé de direction $\dot{\tilde{E}}$.

L'un des apports possibles du modèle "macromicro" que l'on va exposer ici sera la possibilité de donner des éléments physiques de choix entre les expressions (3) et (4) par exemple : en effet la forme du $\widetilde{x}$ que l'on pourrait retrouver n'est pas définie à priori par le modèle, mais découle de l'organisation de la structure cristalline introduite.

Le même type de remarque reste valide pour ce qui concerne la distorsion de la surface de charge. On peut l'obtenir dans un modèle macroscopique soit en modifiant la distance utilisée à l'aide d'un tenseur d'ordre quatre $|9|$ :

$$
J *(\tilde{\Sigma}-\tilde{X})=\left(1.5 \tilde{\tilde{A}}:\left(\tilde{\Sigma}^{\prime}-\tilde{X}^{\prime}\right):\left(\tilde{\Sigma}{ }^{\prime}-\tilde{X}^{\prime}\right)\right)^{0.5},
$$

soit en remplaçant la variable $R$ par un tenseur d'ordre deux, $\widehat{R}|10|$. Les identifications de ces modèles sont alors complexes (que mettre dans $\overrightarrow{\tilde{A}}$, quelle loi d'évolution pour $\widetilde{R}$ ?), et l'on peut s'appuyer sur les théories de lignes de glissement pour évaluer les évolutions de la surface seuil.

Autour du squelette constitué par les variables d'écrouissage, représentant la contrainte interne, on peut construire ensuite des modèles plastique ou viscoplastique. Dans le cas viscoplastique qui nous intéresse ici, on choisit de définir des équipotentielles homothétiques à la surface de charge, ce qui amène à décrire la vitesse de déformation viscoplastique $\widetilde{E}_{\mathrm{v}}$ sous la forme :

$$
\begin{aligned}
& \dot{\tilde{E}}_{\mathrm{v}}=\dot{\mathrm{v}} \cdot \tilde{n} \\
& \text { avec } \dot{v}=\left\langle\frac{J(\tilde{\Sigma}-\tilde{x})-R-k}{K}\right\rangle^{n}
\end{aligned}
$$




\section{Le passage micro-macro}

\section{II.1. Le schéma proposé}

La recontruction du comportement du polycristal à partir des déformations sur les systèmes élémentaires n'est bien sûr pas une entreprise récente. Une première classe de modẻles ne fait pas intervenir directement la microgéométrie du matériau $|11||12|$, il s'agit de généralisation de la théorie des potentiels multiples de Koiter $|13|$. Les développements les plus récents dans le domaine concernent les modèles autocohérents $|14|,|15|$, $|16|$, dans lesquels on cherche au contraire à réaliser le passage monocristal- polycristal. Dans ces modèles, issus de la théorie de Kröner $|17|$, chaque grain $g$ est modélisé séparément, et l'évolution des champs de contrainte et de déformation locaux peut être caractérisée par une relation incrémentale du type :

$$
\dot{\tilde{\sigma}}^{g}=\dot{\tilde{\Sigma}}+\tilde{\tilde{L}}^{*}\left(\dot{\tilde{E}}_{p}-\dot{\tilde{\varepsilon}}_{p}^{g}\right)
$$

Telle quelle, cette expression est difficilement exploitable, car le calcul de $L^{*}$ nécessite la résolution d'une équation implicite (description du comportement d'une inclusion dans un milieu infini homogénéisé). Dans le cas d'une inclusion sphérique, et si le comportement du polycristal est isotrope, on peut remplacer ce tenseur par une fonction scalaire dépendant de la courbe d'écrouissage du matériau et du coefficient de Poisson $|16|$.

Dans la suite, on utilisera à la place de $\widetilde{L}^{*}$ une simple constante $A$ et on travaillera directement sur les quantités intégrées. L'équation devient alors :

$$
\tilde{\sigma}^{g}=\tilde{\Sigma}+A\left(\tilde{E}_{p}-\tilde{\varepsilon}_{p}^{g}\right)
$$

A ce niveau se pose encore le problème du choix des sytèmes actifs. Cette opération est délicate en plasticité, car elle oblige à résoudre un système dont la dimension est égale au nombre de systèmes total, et qui n'est linéaire que par morceaux, dans la mesure où il $n^{\prime} y$ a ni apparition de nouveaux systèmes actifs ni désactivation $|18|$.

C'est pourquoi dans la suite on décide de travailler en viscoplasticité. On va voir que dans ce cas le problème du nombre de systèmes actifs ne se pose plus dans la mesure où l'on peut calculer la vitesse de glissement sur chaque système en fonction de l'état actuel de la cission et des contraintes internes sur ce système : en particulier, on n'a pas besoin des vitesses de contraintes, comme c'est le cas en plasticité. On note que l'on utilise la forme intégrée de la relation de passage entre les grandeurs microscopiques et macroscopiques, (expression (7) et non (6)), car cela permet des simplifications au niveau de l'algorithme. La relation employée ne sera donc qu'une relation approchée en chargement non proportionnel. Il faut noter que le problème qui rend l'utilisation de la forme incrémentale délicate est la connaissance de l'incrément de contrainte macroscopique. Comme cela sera discuté plus loin, on le rencontre essentiellement pour les chargements à déformation imposée. C'est pourquoi il n'apparait pas dans la littérature car les versions viscoplastiques des modèles autocohérents sont étudiées en fluage stationnaire $|19|,|20|,|21|$.

Le schéma général de la figure 1 résume les différentes étapes d'un calcul pas à pas réalisé avec le modèle. On décrit ici un algorithme purement explicite dans lequel la connaissance de l'état de contrainte (étape 3) et des variables d'écrouissage (étape 5 ) suffit à déterminer la vitesse d'écoulement et l'évolution de l'écrouissage. On peut travailler en contrainte ou déformation imposée sur chacune des six composantes du tenseur, l'étape 2 permettant dans tous les cas de retrouver la contrainte macroscopique présente en début d'incrément. On note que le fait d'utiliser la relation 4 sous forme intégrée permet de continuer à travailler en mode explicite, alors que son écriture sous forme dérivée ferait intervenir le terme $\dot{\Sigma}$ que l'on ne peut calculer qu'après l'étape 11 dans le cas de chargements imposés en déplacement; d'un autre côté, il ne serait pas sain à ce niveau d'utiliser la valeur obtenue au pas précédent, car on induirait alors des erreurs à chaque point anguleux du chargement (inversion par exemple). Les lois de comportement, qui vont être discutées dans la suite, relient la vitesse de glissement sur chaque système de glissement avec la cission résolue et les variables d'écrouissage supposées connues sur ce même système. On n'a fait ici que reproduire les expressions unidimensionnelles des relations macroscopipques présentées au chapitre I. La reconstruction de la déformation macroscopique s'effectue ensuite classiquement en deux étapes, sur tous les systèmes d'un grain donné (étape 8a) et sur l'ensemble des grains (étape 9). On note que pour obtenir la vitesse de déformation $d u$ grain $g$, on utilise la partie symétrique du produit dyadique $\bar{n}^{s} \bar{i}^{\mathbf{s}}\left(\bar{n}^{\mathrm{s}}\right.$ normale au plan; $\bar{i}^{\mathbf{s}}$ direction de glissement), que l'on désigne par $\tilde{m}^{\$}$ et que l'on donne un certain poids $w^{5}$ aux contributions de chacun des systèmes. Ce poids correspond au pourcentage de systèmes actifs selon un mécanisme donné $|33|$ : il est clair en effet qu'en chargement complexe il faut différencier la contribution du glissement sur un système pour l'écrouissage et pour la déformation. C'est ainsi que le choix d'une valeur uniforme comprise entre 0 et 1 pour tous les systèmes permettra de séparer l'effet de toutes les dislocations qui contribuent à l'écrouissage, de l'effet de dislocations mobiles, moins nombreuses, qui contribuent réellement au glissement. Par ailleurs, la transformation de $w^{\mathbf{S}}$ en une variable peut devenir intéressante si plusieurs types de systèmes de glissement sont actifs : elle permettrait en effet de mieux contrôler les activations et désactivations au cours d'un chargement cyclique par exemple |34|. Par ailleurs, la sommation sur l'ensemble des grains (étape 9) se fait de la façon habituelle, en attachant une fraction volumique à chacun d'entre eux, ce qui permet de définir la texture. On peut adjoindre à cette méthode un calcul de pas automatique (étape 11) à l'ordre 1 que l'on obtient au niveau macroscopique en comparant les dérivées première et seconde de la déformation viscoplastique (la dérivée seconde étant estimée en fonction des valeurs antérieure et actuelle de la dérivée première, et du pas de temps précédent).

L'algorithme utilisé nécessite relativement peu d'espace en mémoire centrale. A un instant donné, seules doivent être conservées pour le pas suivant, en plus des grandeurs macroscopiques, les valeurs des variables d'écrouissage microscopique $x^{\mathbf{S}}$ et $r^{\mathbf{S}}$ et celles de la déformation viscoplastique du grain, soit deux fois le nombre de systèmes total, plus six fois le nombre de grains, soit encore trente fois le nombre de grains pour un métal cfc, à raison de douze systèmes par grain.

Nous allons maintenant discuter un peu plus en détail les expressions des lois de comportement proposées au niveau microscopique. 


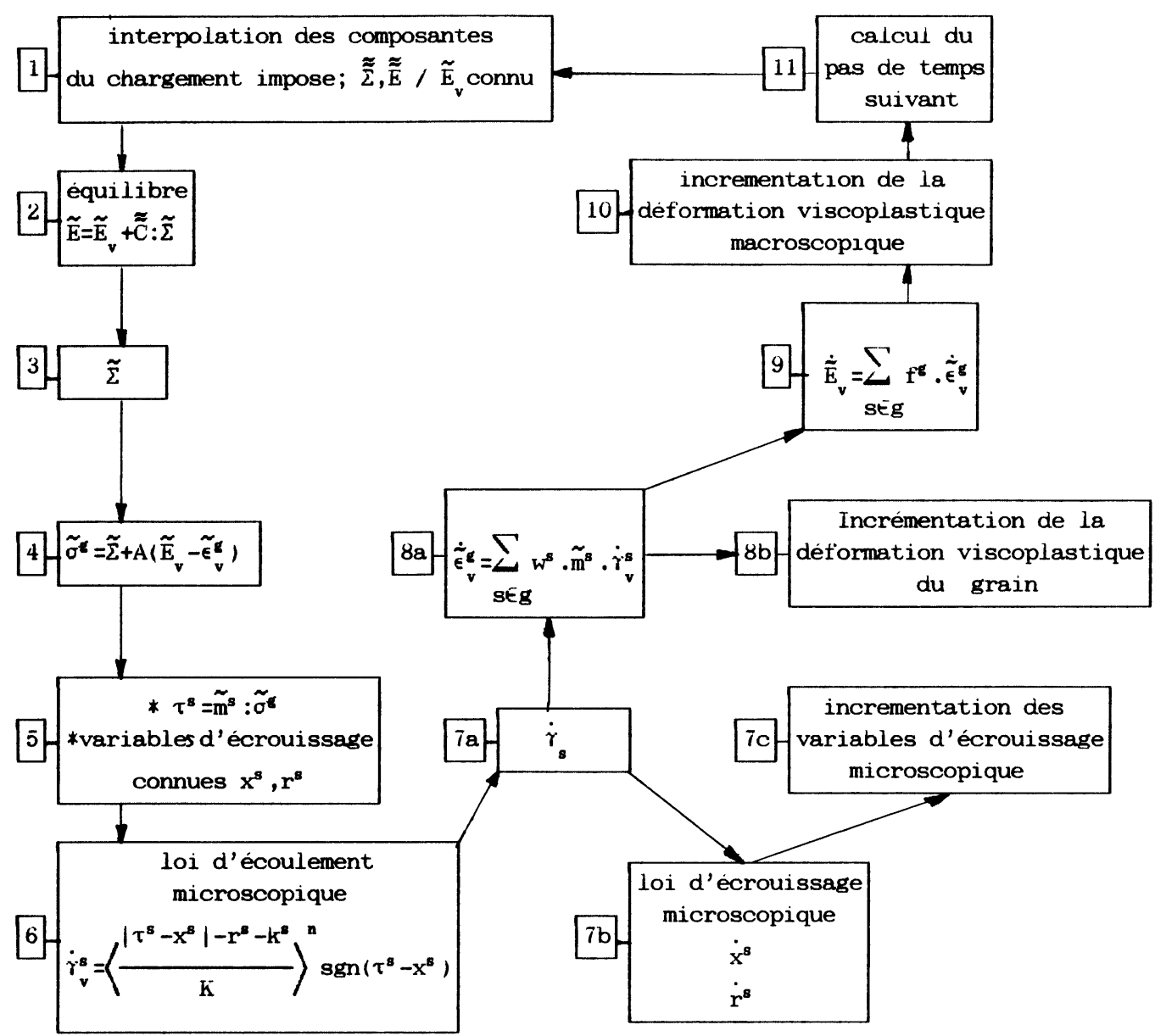

$\langle f\rangle=0$ si $f\langle 0,\langle f\rangle=f$ si $f\rangle 0$

$$
\begin{aligned}
& \text { Figure_1 - Le schéma général de la méthode proposée. } \\
& \text { [Fig. 1 - The algorithm used for the numerical simulation.] }
\end{aligned}
$$

\section{II.2. Ecriture de l'écrouissage au niveau micro-} scopique

On peut facilement inverser la relation qui est proposée à l'étape 6 de la figure 1, pour obtenir, en présence d'écoulement viscoplastique, une partition de la contrainte en une contrainte interne et une partie contrainte visqueuse, $\tau_{\mathrm{v}}{ }^{\mathrm{s}}$ :

$$
\tau^{s}=x^{s}+\left(r^{s}+k^{s}+\tau_{v} s\right) \text {. signe }\left(\dot{\gamma}_{v}^{s}\right)
$$

où

$$
\tau_{v}^{s}=K \mid \dot{\gamma}_{v} s^{1 / n}
$$

Cette décomposition est illustrée sur la figure 2, dans le cas où l'on choisit un écrouissage cinématique linéaire pour $x^{\mathbf{s}}$. On obtient ainsi les deux valeurs limite de $\tau^{\mathrm{cs}}$, cission critique sur le système $s$ :

$$
\begin{aligned}
& \tau^{\mathbf{c s +}}=\mathbf{x}^{\mathbf{s}}+\mathbf{r}^{\mathbf{s}}+\mathrm{k}^{\mathbf{S}} \text { en "traction" } \\
& \tau^{\mathbf{c s}-}=\mathbf{x}^{\mathbf{s}}-\mathbf{r}^{\mathbf{s}}-\mathrm{k}^{\mathbf{S}} \text { en "compression" }
\end{aligned}
$$

Cette distinction entre les comportements selon le sens de sollicitation permet de ne considérer qu'un seul système de glissement pour une direction donnée, qui sera activable dans les deux sens. Cela implique bien sûr un certain nombre de restrictions sur les interactions entre systèmes d'un même grain : ainsi par exemple on fige l'écrouissage latent entre deux systèmes situés sur une même direction, mais on va voir qu'il reste encore suffisamment de paramètres libres dans le modèle pour que cela ne pose pas de problème particulier.

On fait l'hypothèse que les interactions entre les systèmes de glissement portent uniquement sur la partie isotrope de l'écrouissage. Cela correspond au fait que l'on attribue habituellement $1^{\prime}$ effet Bauschinger aux interactions dislocations-précipités. Il s'agit donc d'un type de durcissement où chaque système réagit individuellement avec son envionnement. Le phénomène a été étudié en détail dans les alliages durcis par précipités (voir par exemple les revues les plus récentes $|22|,|23|$ et $|24|)$. Les résultats obtenus montrent qu'aux très faibles déformations le 


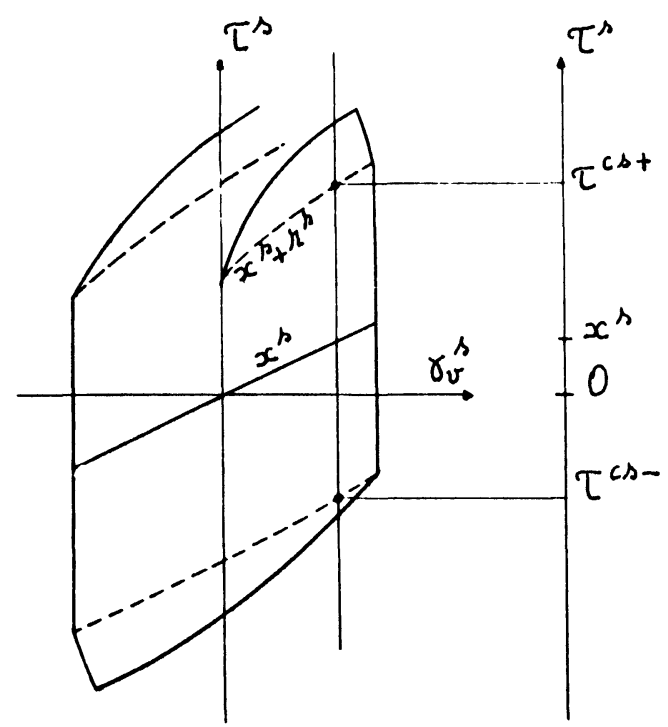

Figure 2 - Une boucle d'hystérésis typique obtenue en traction-compression au niveau d'un système de glissement.

Fig. 2 - A typical stressustrain loop under tension] compression loading, as seen by a slip system.

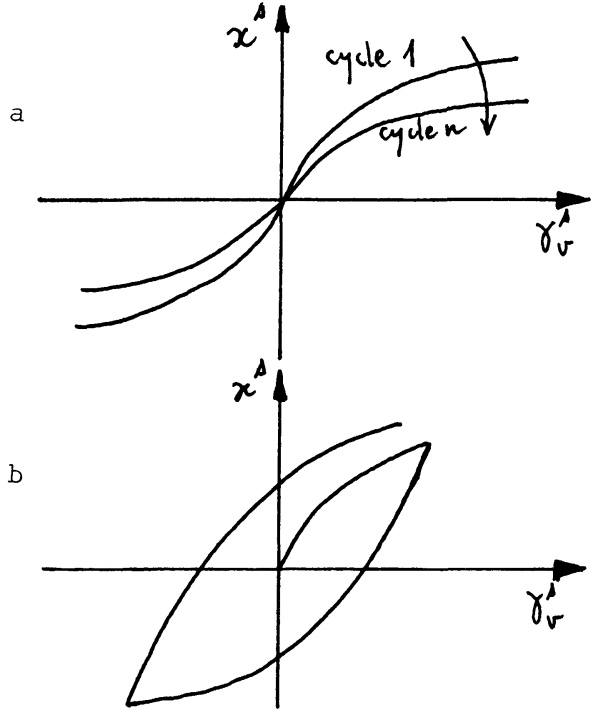

Figure 3 - Les différents types d'écrouissage cinématique.

a. Cinématique non linéaire sans hystéresis.

b. Cinématique non linéaire avec hystéresis.

Fig. 3 - Various kinematic hardening types :

a. Non Linear kinematic hardening without hysteresis;

b. Non Linear kinematic hardening with hysteresis.]

On définit l'écrouissage isotrope dans un grain par un vecteur dont la dimension correspond au nombre de systèmes ( 12 pour un cfc, par exemple). $L^{\prime}$ interaction est introduite entre les différents systèmes d'un même grain par l'intermédiaire d'une matrice d'écrouissage $\widetilde{H}^{*}$ portant sur les variables de glissement viscoplastique cumulé $v^{*}$ On choisit pour cette matrice une forme qui génêralise l'égalité (2), avec pour les mêmes raisons que dans le cas macroscopique une saturation de cet écrouissage avec $\mathrm{v}^{\mathrm{s}}$.

$$
\begin{aligned}
& \dot{x}^{s}=C \dot{\gamma}_{v}^{s} \\
& \dot{x}^{s}=C \cdot \exp \left(-D_{1} \cdot \gamma_{v}^{s}\right) \cdot \exp \left(-D_{2} v^{s}\right) \cdot \dot{\gamma}_{v}^{s} \\
& \dot{x}=C \dot{\gamma}_{v}^{s}-D x^{s} \cdot \dot{v}
\end{aligned}
$$

$C, D, D_{1}$ et $D_{2}$ sont des coefficients caractéristiques du matériâu.

L'utilisation de l'expression (10a) revient à introduire un écrouissage cinématique linéaire sur chaque système de glissement, l'expression (10b), quant à elle, va décrire un écrouissage du type "élasticité non linéaire" donnant naissance à des boucles d'hystérésis globales comportant des points d'inflexions $|26|,|27|$; dans l'expression qui est choisie ici, la première exponentielle permet de décrire la forme voulue de la boucle, alors que la seconde conduit à l'effacement de ce type d'écrouissage cinématique pour de grandes amplitudes de déformations, ou après quelques dizaines de cycles pour les faibles amplitudes. La dernière expression proposée (égalité 10c) est la forme unidimensionnelle de l'expression (3) et conduit donc à une variable $x^{s}$ qui décrit elle même une boucle d'hystérésis. La figure 3 schématise les deux dernières formes d'écrouissage, alors que la première forme était représentée en figure 2.

$$
\begin{gathered}
\dot{\bar{r}}=\tilde{H}^{*} \cdot \dot{\bar{v}} \\
H_{r s}^{*}=b \cdot Q \cdot H_{r s} \cdot e^{-b v^{s}}
\end{gathered}
$$

avec

La valeur initiale de $r^{\mathbf{S}}$ sur chaque système de glissement vaut $k^{\mathbf{s}}$, que $l$ 'on peut prendre uniforme sur tous les sytèmes de même type; par ailleurs l'augmentation de l'écrouissage sur un système donné sera dépendant du nombre de systèmes actifs dans le grain, le système d'indice $s$ détenant un pouvoir d'écrouissage (augmentation de la cission résolue critique) de $\left(H_{r} \cdot Q\right)$ sur le système $r$. Avec un tel type de fonctionnement, on sera donc capable de décrire des durcissements plus grands en chargement complexe qu'en chargement simple (plus de systèmes activés) ou bien encore l'effet de mémoire du trajet de déformation sous chargement uniaxial (la réalisation d'un préécrouissage à un fort niveau de déformation augmente de façon importante le durcissement aux faibles niveaux).

Dans la suite de l'exposé, on va maintenant illustrer le fonctionnement du modèle sur des essais réels, en choisissant une description simple de la microstructure. 


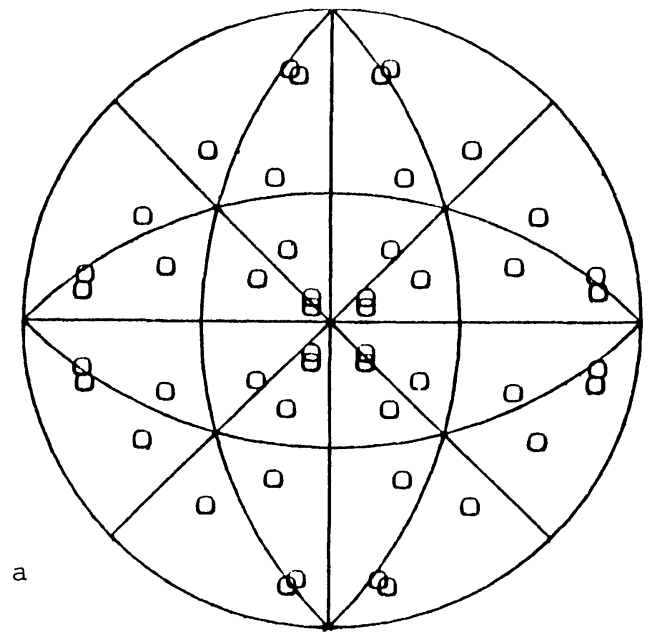

III. Utilisation du modèle

\section{III.1. Description de la microstructure}

On cherche ici à reconstruire avec le moins de grains possible un matériau cfc qui soit le plus isotrope possible en chargement tridimensionnel. On choisit donc de respecter au moins la symétrie cubique et la méthode choisie pour cela consiste à positionner chaque grain de maniére à ce que les trois axes du "repère du laboratoire" soient équivalents pour lui. Cela conduit à des relations particulières entre les trois angles d'Euler $\varphi, \phi$, $\varphi$ (notation de Bunge |28|) qui font passer du "repère du laboratoire" au repere cristallographique. On suppose pour les obtenir que les colonnes de la matrice de passage peuvent être déduites l'une de l'autre par permutation circulaire, il vient alors :

$\varphi_{1}=\frac{\pi}{2}+\varphi_{2}$

$\phi=\operatorname{Arc} \cos \left(\cos \varphi_{2} \cdot \sin \varphi_{2} /\left(1-\cos \varphi_{2} \cdot \sin \varphi_{2}\right)\right)$

On génère ainsi une famille de 48 grains que l'on installe en position de glissement simple pour les expériences de traction. La figure 4 indique la position des axes $X, Y$ et $Z$ du repère du laboratoire dans le repère cristallographique en choisissant comme pôle 001 . La figure 5 , quant à elle, montre le résultat d'essais de traction et de cisaillement selon différentes directions : on $y$ constate que les tractions selon les axes révelent un écrouissage plus faible que pour les deux autres tractions, pour lesquelles plus de systèmes sont activés. Les cisaillements déclenchent eux aussi un nombre important de systèmes, si bien que les contraintes équivalentes atteintes sont un peu trop élevées. Il reste que le matériau obtenu présente une symétrie cubique parfaite et l'on s'accomodera de l'écart enregistré ici, dont on pourra tenir compte dans les identifications.

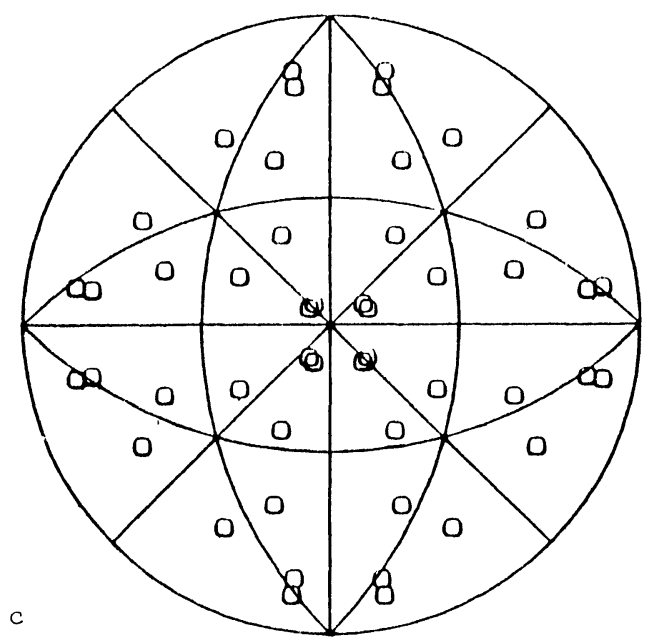

Figure 4 - Projections stéréographiques des axes $X, Y$ et $Z$ du repère fixe dans le repère cristallographique (pôle 001 ).

Fig. 4 - Inverse pole figures showing $X, Y$ and $Z$ axis in crystallographic coordinate axis.

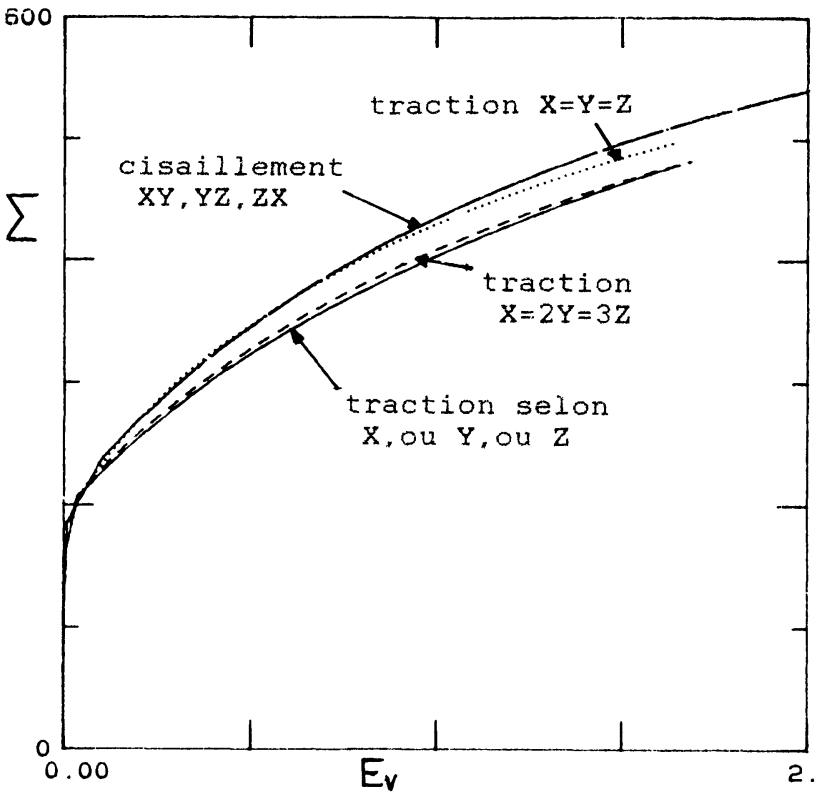

Figure 5 - Comparaison des courbes d'écrouissage en traction et torsion pour différentes directions (contraintes et déformations équivalentes de Misès).

Fig. 5 - Comparison between tensile and shear monotonic curves for various loading directions (Mises type equivalent are chosen for stress and Llastic strain). 


\section{III.2. Présentation des essais réalisés}

On veut modéliser des essais dont la description détaillée est donnée par ailleurs $|29|$. Ces essais permettent de confirmer l'augmentation d'écrouissage liée à la non-proportionnalité du chargement, qui a été mis en évidence il y a une dizaine d'année $|30|$, et qui a été très étudiée depuis (voir par exemple dans $|31|$ une étude systématique de p? usieurs modéles macroscopiques possibles). Ils ont été réalisés à l'ONERA sur une machine de traction-torsion hydraulique asservie. Les spécimens étudiés sont des tubes minces en acier inoxydable austénitique 17-12 SPH (type 316L), sollicités à angle et déplacement imposés, à température ambiante. La courbe d'écrouissage cyclique (fig. 6) résume l'effet de durcissement supplémentaire rencontré pour différents types de chargement; les niveaux obtenus se situent entre 200 et $300 \mathrm{MPa}$ au dessus de la courbe d'écrouissage de référence pour des essais où angle et déplacement sont déphasés de $90^{\circ}$, pour des trajets en forme de croix ou de carrés en traction-cisaillement, ou bien encore pour un essai où l'on applique successivement 10 cycles de torsion et 10 cycles de traction, aux mêmes niveaux de déformation équivalente. Dans la suite, on va illustrer le fonctionnement du modèle en identifiant les paramètres pour représenter correctement les essais en chargement proportionnel (traction ou torsion) et en l'appliquant ensuite sur un essai en chargement hors phase $90^{\circ}$ et au chargement en $X$. Les données expérimentales correspondant à ces deux derniers essais sont reportées respectivement en figure 7 et 8 . On notera qu'en raison de la longueur de l'éprouvette le chargement imposé local sur la partie utile, mesuré par jauges, ne reproduit pas fidèlement le déphasage appliqué sur les têtes de l'éprouvette (fig. 7a, fig. 8b). On appliquera bien sûr le chargement local pour effectuer les simulations numériques. Afin de mettre en évidence le durcissement supplémentaire obtenu dans ces essais, on a reporté en fig. $7 \mathrm{~b}$ les références que l'on obtient en traction ou en torsion simple pour des niveaux de déformation équivalents. Une simulation avec un modèle classique dornerait un cercle passant par ces références. De la même manière, on a reporté la simulation de l'essai en " $X$ " effectuée avec le modèle macroscopique présenté au chapitre I (fig. 9) : on constate que l'allure des boucles est respectée, mais pas le niveau de contrainte. Comme on l'a signalé au départ, il est possible d'améliorer cette description en modifiant le modèle macroscopique $|31|$, mais on va voir que le modèle que l'on propose conduit également, de façon naturelle à des ordres de grandeur corrects à la fois pour les chargements proportionnels et les chargements non proportionnels.

\section{III.3. Identification du modèle}

Les coefficients à identifier sont :

- le coefficient A de l'équation (7), correspondant à un écrouissage cinématique lié aux incompatibilités de déformation entre grains;

- les coefficients $C$ et $D$ caractérisant l'écrouissage cinématique à l'intérieur du grain (on choisit ici l'égalité (10c);

- pour la description de l'écrouissage isotrope à l'intérieur du grain (égalité (11), la valeur $r$ du cisaillement critique initial $\mathrm{k}^{\mathbf{s}}$ sur chaque système ( $s=1 \ldots 12), l^{\prime}$ augmentation d'écrouissage possible $Q$ et le coefficient définissant la vitesse de saturation b. En l'absence d'indications précises pour l'alliage considérer, on choisit des valeurs égales pour l'auto-écrouissage et l'écrouissage croisé $\left(\forall i, \forall j, H_{i j}=1\right)$;

- les coefficieñts $\dot{K}$ et $n$ (égalité (8b)), qui caractérisent la viscosité du matériau.

Les termes de pondération $w^{s}$ (cadre $8 a$ dans la fig.1) sont pris égaux à $1 / 3$, les fractions volumiques de tous les grains sont égales.

Huit coefficients sont donc nécessaires pour décrire le comportement viscoplastique. Dans le cas qui nous intéresse, on va chercher à effectuer une identification de type "plastique", donc dans laquelle on travaille avec une contrainte visqueuse relativement faible. On choisira pour cela une valeur faible pour $K$ et grande pour $n$, ce qui donne une faible influence de la vitesse de déformation sur les simulations effectuées. D'un autre côté, on ne s'intéresse pour l'instant qu'au cycle stabilisé, on va donc accélérer la stabilisation numérique en choisissant une valeur élevée pour b. Les valeurs utilisées pour l'ensemble des simulations sont reportées dans le tableau $I$.

On trouvera sur les figures 10 a 12 les simulations numériques effectuées. Les coefficients sont prioritairement ajustés sur l'essai de tractioncompresssion car, ainsi que le montre la figure 5, l.e fait d'utiliser une description réduite de la microstructure conduit à une surévaluation de la contrainte en torsion. On constate que l'on retrouve bien la très forte augmentation d'écrouissage dans l'essai hors phase, (fig.11), et que les niveaux et la forme des boucles obtenues pour le chargement en " $X$ " sont correctement représentés, (fig.12), contrairement à ce qui se passe pour le modèle macroscopique classique ( $\mathrm{fig.9}$ ).

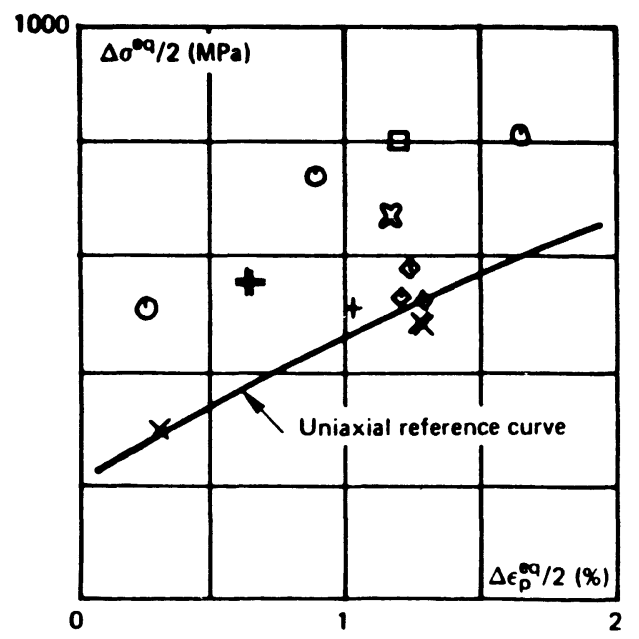

Figure 6 - Influence du trajet de chargement sur la courbe d'écrouissage cyclique (inox 17-12 SPH, température ambiante) $|29|$.

+ traction-compression; $x$ torsion; traction-torsjon en phase;O口X traction- torsion hors phase $90^{\circ}$, trajet carré, en $x$; $\neq$ traction-torsion en séquences.

Fig. 6 - Influence of the loading path on the cyclic hardening curve (316L stainless steel, room temperature ) $|29|$.

+ tension; $x$ torsion; in phase tension-torsion; out of phase $\left(90^{\circ}\right)$ tension-torsion; $\square$ complex paths; $\neq$ sequential tension and torsion loadings. 


\begin{tabular}{|c|c|c|c|c|c|c|c|}
\hline$A$ & $C$ & $D$ & $r_{0}$ & $Q$ & $b$ & $K$ & $n$ \\
\hline 3000 & 1000 & 50 & 50 & 50 & 500 & 50 & 25 \\
\hline
\end{tabular}

Tableau I - Les coefficients utilisés pour l'ensemble des simulations. (unités : s, MPa).

Table_I - The coefficients used for numerical simulations (urits : s, MPa).
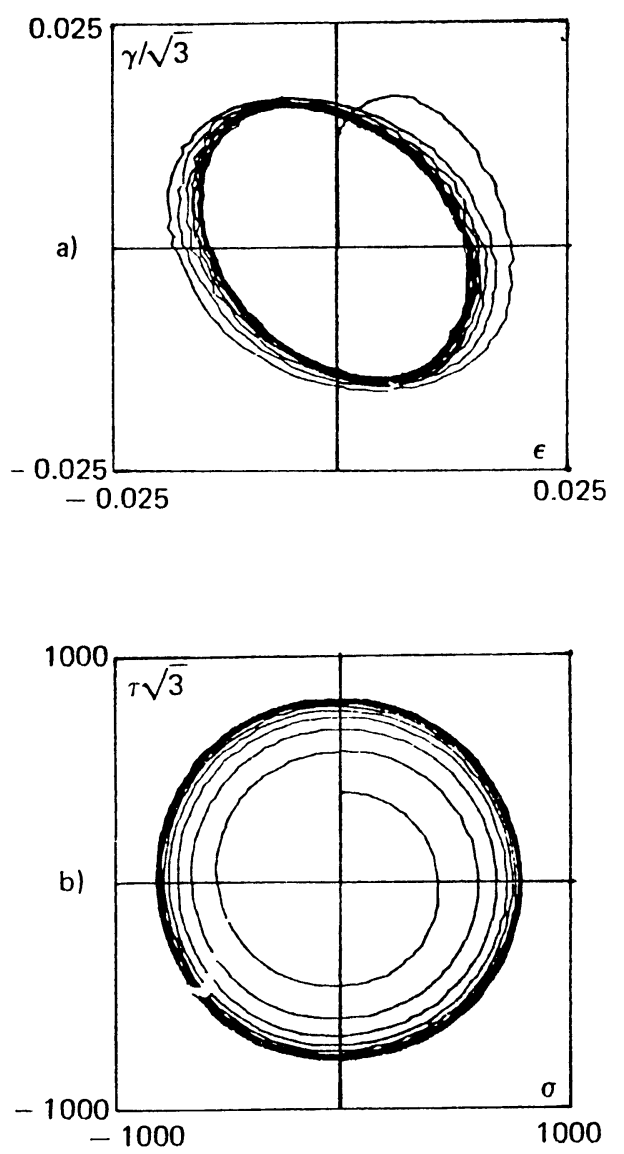

Figure 7 - Essai à déplacement et angle imposés, déphasage. $90^{\circ}$.

a. Chargement imposé local en $\varepsilon-\gamma / \sqrt{3}$.

b. Réponse en $0-\tau \sqrt{3}$.

[Fig. 7 - Test with controlled displacement and] angle (90 phase lag).

a. Local imposed loading, $\varepsilon-\gamma / \sqrt{3}$;

b. Stress response, $\sigma-\tau \sqrt{3}$.

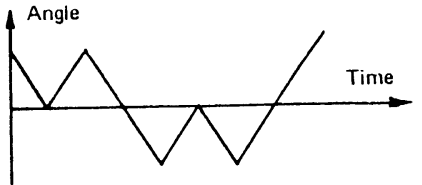

a
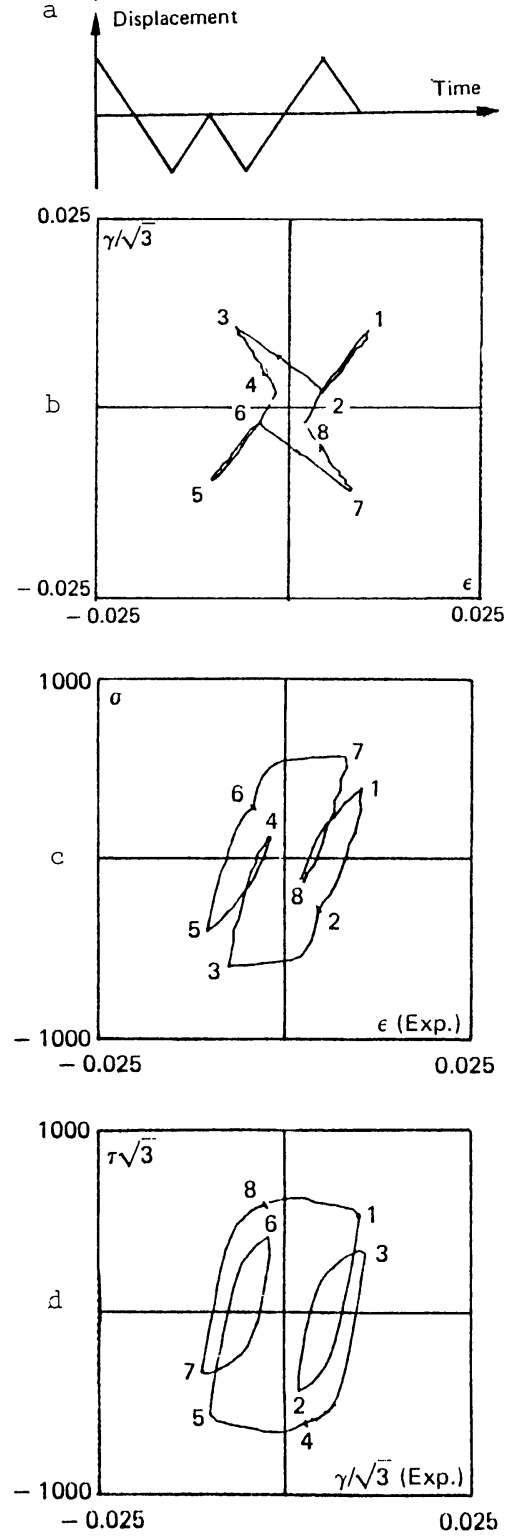

Figure 8 - Chargement en "X" en angle et déplacement.

a. Chargement imposé en fonction du temps.

b. Chargement imposé local en $\sigma-\gamma / \sqrt{3}$.

c. Boucle d'hystérésis experimentale en chargement axial, $\sigma-\varepsilon$.

d. Boucle d'hystérésis expérimentale en cisaillement, $\tau \sqrt{3}-\gamma / \sqrt{3}$.

Fig. 8 - "X" shape loading in angle and displacement space.

a. Imposed loading vs.time;

b. Local imposed loading, $\varepsilon-\gamma / \sqrt{3}$;

c. Experimental loop, axial components, $\sigma-\varepsilon$;

d. Experimental loop, shear components, $\tau \sqrt{3}-\gamma /$

d. $\sqrt{3}$. 

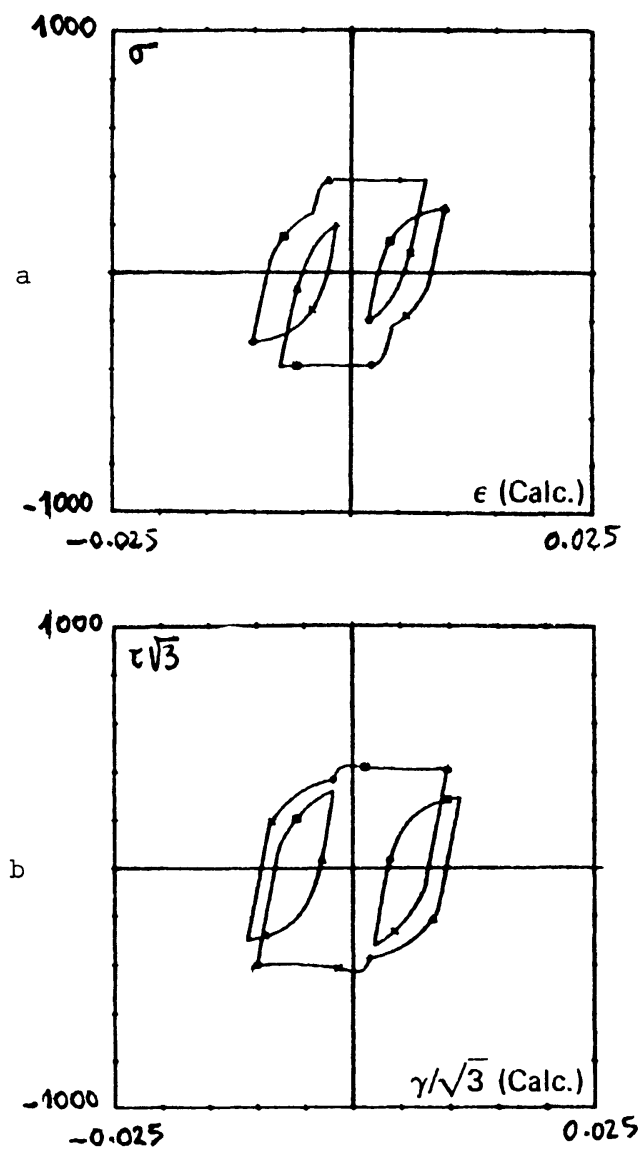

Figure 9 - Chargement en "X" en angle et déplacement, simulation avec un modele macroscopique classique.

a. Boucle d'hystérésis calculée en chargement axial, $\sigma-\varepsilon$.

b. Bouclé d'hystérésis calculée en cisaillement, $-\gamma$.

Fig. 9 - "X" shape loading path, numerical simulation with a classical macroscopic model.

a. Predicted loop, $\sigma-\varepsilon$;

b. Predicted loop, $\tau \sqrt{3}-\gamma / \sqrt{3}$.
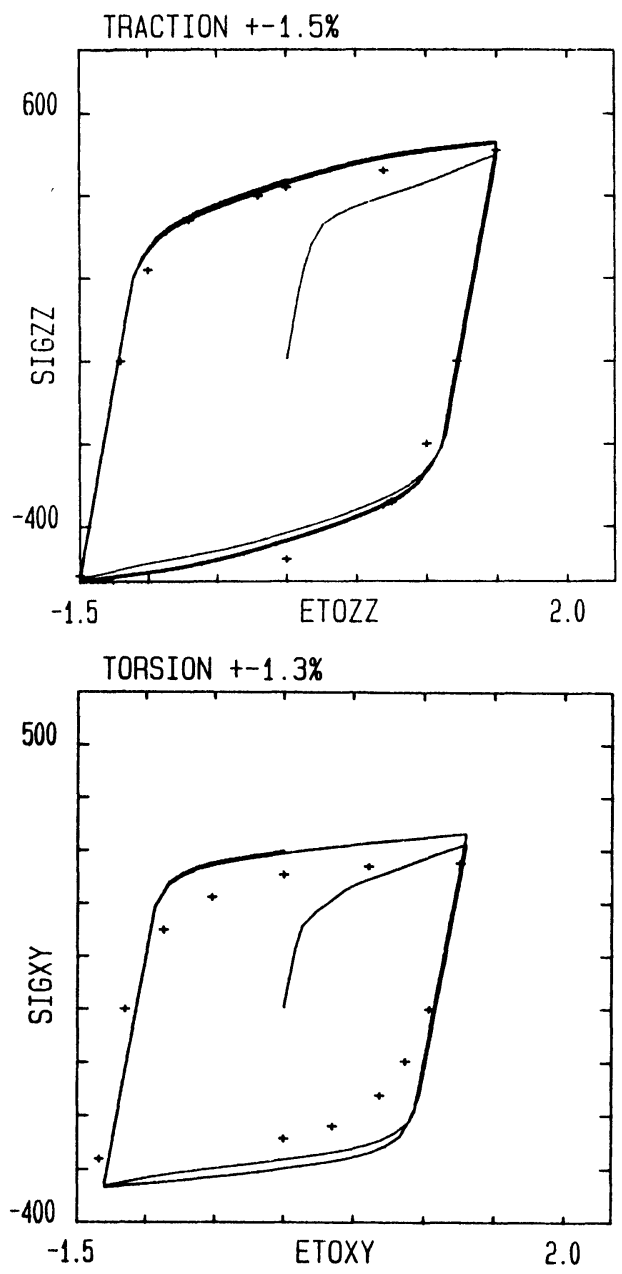

Figure 10 - Simulation numérique des essais de référence en chargement proportionnel (exp : +). a. Traction-Compression $\pm 1,5 \%$.

b. Torsion $\pm 1,3 \%$.

Fig. 10 - Numerical simulation of the reference onedimensionnal tests (exp : + ).

a. Tension-Compression $+1.5 \%$;

b. Torsion $+1.3 \%$.

HORS PHASE 68.

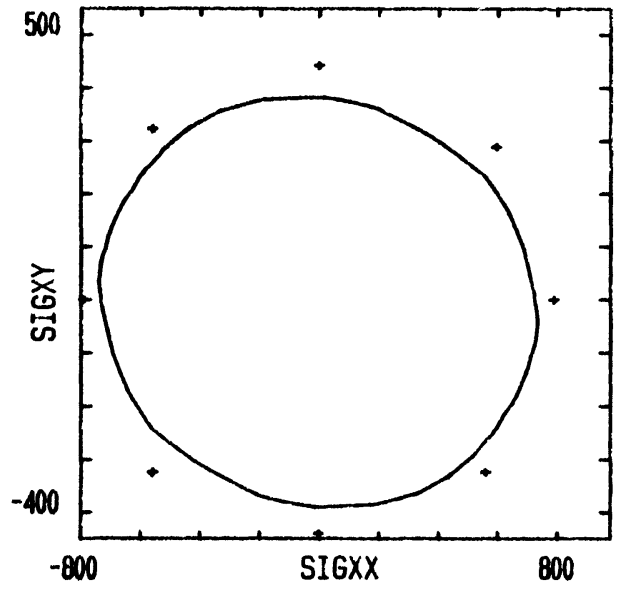

Figure 11 - Simulation numérique du chargement

[Fig. 11 - Numerical simulation of out of $p$. se test.] 
CHARGEMENT $X$

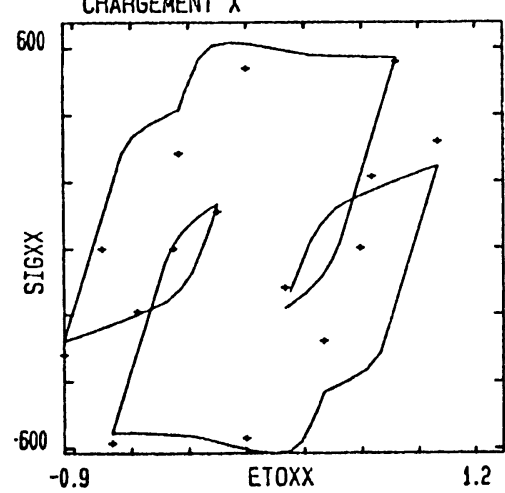

CHARGEMENT $X$

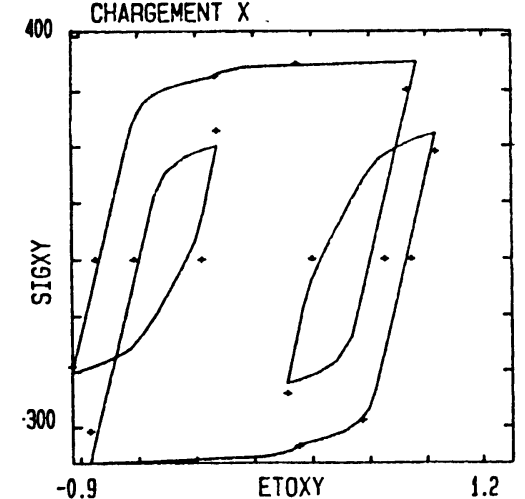

Figure 12 - Simulation numérique du chargement en $\overline{\mathrm{X}}$.

a. Boucle contrainte-déformation en chargement axial (XX).

b. Boucle contrainte-déformation en cisaillenent $(X Y)$.

Fig. 12 - Numerical simulation of "X" shape loading] path.

a. Predicted loop, $\sigma-\varepsilon$

b. Predicted loop, $2 \sqrt{3}-\gamma / \sqrt{3}$.

IV. Discussion

Dans ce chapitre, on illustre le fonctionnement du modelle en traçant l'évolution du nombre de systèmes actifs en fonction du chargement appliqué. On montre que le modele représente correctement l'effet de mémoire $|32|$. On montre aussi par un exemple que le modéle prévoit des durcissements qui $n$ 'ont pas encore été mis en évidence dans le cas de chargements tridimensionnels.

\section{IV.1. L'effet de mémoire}

Lors d'un chargement unidimensionnel, l'effet de mémoire se manifeste par le fait que la valeur de l'écrouissage obtenu dépend de la déformation plastique maximale atteinte. La figure 13 illustre ce type de phénomène, simulé par le modẻle avec les coefficients du tableau I. On y trouve la simulation d'un essai de référence à $\pm 0,5 \%$ ( $\mathrm{fig} .13 \mathrm{a}$ ), suivie du calcul d'un essai pour lequel on aurait appliqué une déformation de $\pm 1,5 \%$ avant de revenir $a ̀+0,5 \%$. Le niveau obtenu dāns le deuxième cas est plus élevé, et cela s'explique par le fait que le nombre de systèmes qui ont été activés pendant le chargement à $+1,5 \%$ (et qui ont donc contribué à l'écrouissage) est plus élevé qu'à $+0,5 \%$ (voir fig.13c, où sont reportés pour chaqué simulation les systèmes plastifiés, selon la définition du IV.3).
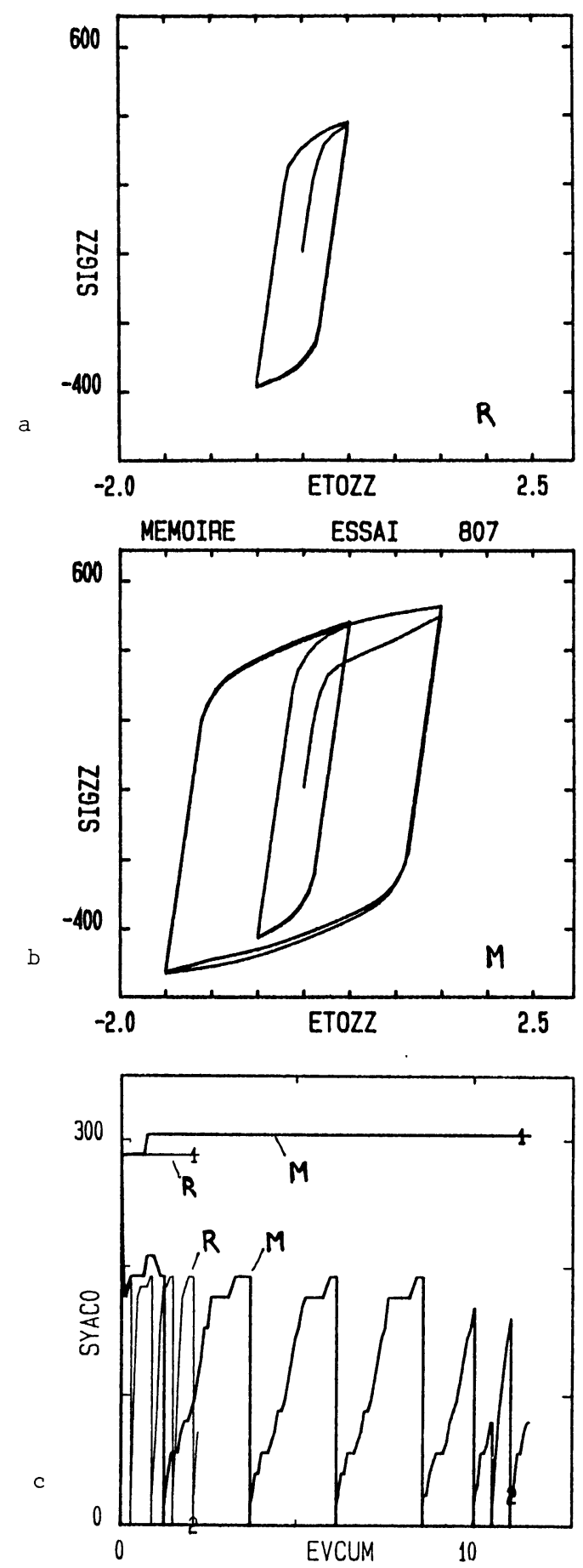

Figure 13 - 1llustration de l'effet de mémoire.

a. Cycle de référence à $+0,5 \%$ (essai $R$ ).

b. Simulation de 2 cycles à $\pm 1,5 \%$, puis $0,5 \%$ (essai M).

c. Le nombre de systèmes plastifiés (Courbes 1) et actifs (courbes 2 ) pour les essais $R$ et $M$.

Fig. 13 - Illustration of strain memory effect] (simulation).

a. Reference test, $\pm 0.5 \%$ (test $\mathrm{R}$ ).

b. Two cycles at $+1.5 \%$, then $+0.5 \%$ (test $M$ );

c. Number of slip̄ system showing non zero plastic shear, tests $\mathrm{R}$ and $\mathrm{M}$, (plastic, curve 1; active, curve 2 ). 

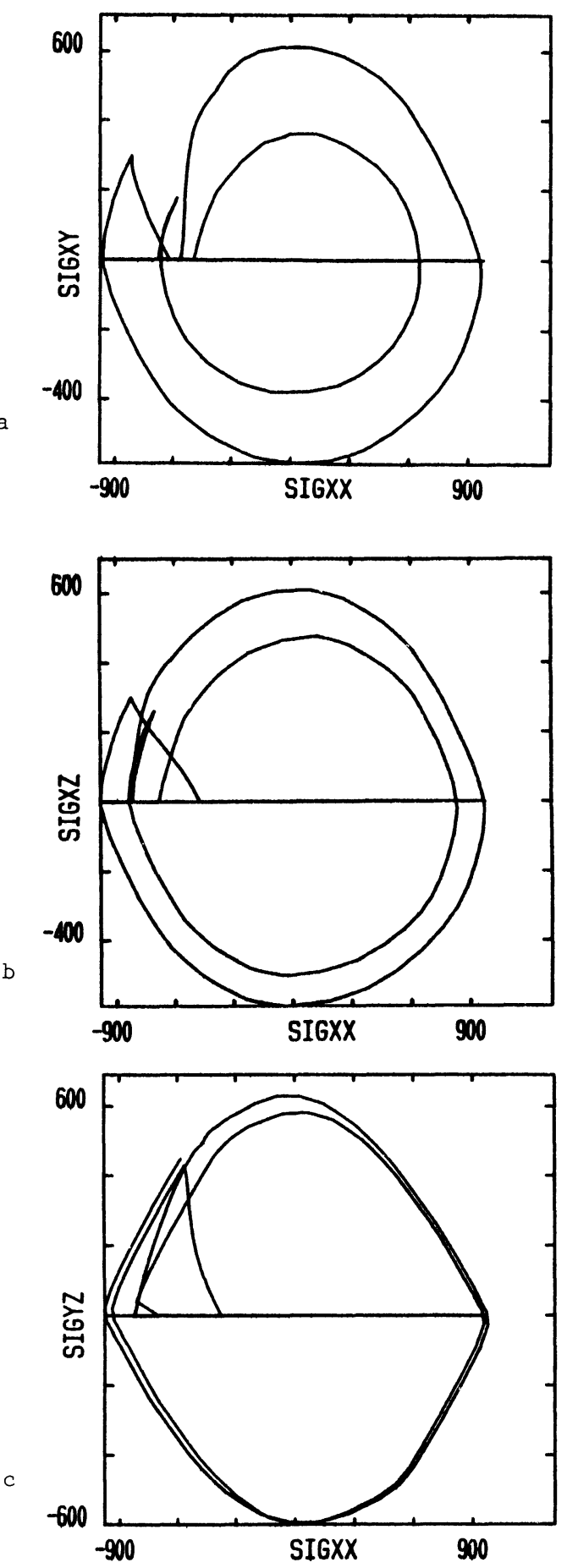

\section{IV.3. Systèmes actifs, systèmes plastifiés}

Les systèmes actifs à un instant donné du chargement sont ceux pour lesquels la vitesse de glissement est non nulle; on appelle ici système plastifié un système qui a été actif au moins une fois depuis le début du chargement; ce système sera donc repéré par une déformation cumulée non nulle. On a reporté sur la figure 15 l'évolution du nombre moyen de systèmes plastifiés et actifs par grain pour une traction simple (courbes 1 et 2 ). On constate que de très nombreux systèmes sont plastifiés, et que le nombre des systèmes actifs diminue : cela est du au fait que, l'écrouissage étant le même sur les systèmes qui glissent peu et les systemes fortement actifs, la cission appliqué sur les premiers finit par être rejoint par la cission critique. Le nombre de systemes élevé qui est concerné par le glissement (si l'on se référe aux résultats habituellement obtenus dans les modéles de plasticité instantanée) ne doit pas faire oublier que dans le cas de la viscoplasticité, peu de systèmes ont une contribution déterminante pour la vitesse de glissement. C'est ce que montrent les courbes 3 et 4 de la figure 15, où $l^{\prime}$ on a introduit un seuil : si l'on ne considère que les systèmes dont la vitesse de glissement actuelle (le cisaillement cumulé actuel) est supérieure au dixième de la vitesse de glissement maximum observée (au dixième du cisaillement cumulé maximum), on obtient alors des valeurs plus classiques, de l'ordre de 3 ou 4 systèmes par grain.

On notera sur la figure 16, qui correspond à l'essai décrit en IV.2 que, dans ce cas, chaque changement de plan introduit de nouveaux systèmes plastifiés, mais que, l'écrouissage augmentant, le nombre de systèmes actifs diminue au cours du chargement.

Enfin, on constate (fig. 17), que le nombre de systèmes actifs obtenu est étroitement lié à la matrice d'écrouissage : si les termes hors de la diagonale sont élevés, l'écrouissage croisé conduit à un blocage rapide sur les directions les moins sollicitées.

hors phase dans trois plans successifs.

a. Plan $x x-x y$;

b. Plan $x x-x z$;

Fig. 14 - Numerical simulation of out of phase loading path in three successive planes.

a. $x x-x y$ plane;

b. $x x-x z$ plane

c. $x x-y z$ plane. 


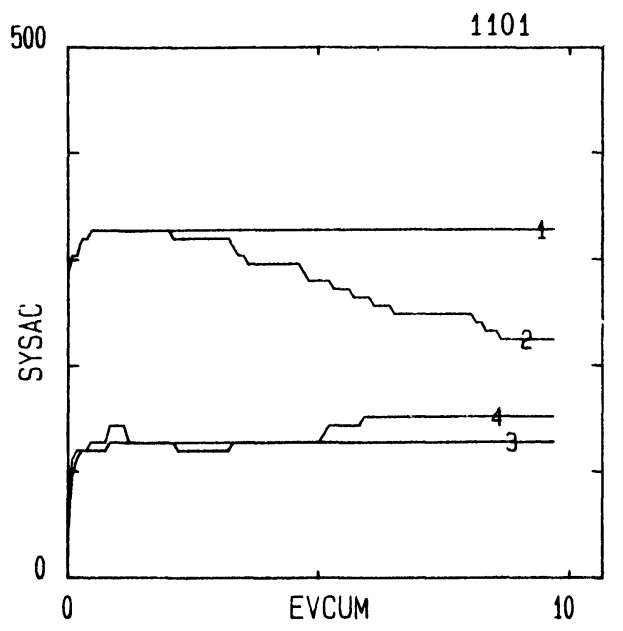

Figure 15 - Evolution du nombre de systèmes concernés par le glissement au cours d'une traction simple.

(1) Systèmes plastifiés $(v>0)$;

(2) Systèmes actifs $\dot{\gamma}_{y} \neq 0$;

(3) Systemes significativement ( $v>0.1 * v_{\max }$ );

(4) Systêmes slgaxificativement actifs $\left(\left|\dot{\gamma}_{v}\right|\right)$ $\left.0.1\left|\dot{\gamma}_{\max }\right|\right)$.

Fig. 15 - Evolution of the number of active systems during a monotonic tension test.

(1) Systems with a positive accumulated shear strain $(\mathrm{v}>0)$;

(2)Active systems $\left(\dot{\gamma}_{v} \neq 0\right)$;

(3) Systems with a significant accumulated shear strain ( $v>0.1 \mathrm{v})$;

(4) Significantly active systems $\left(\left|\dot{\gamma}_{v}\right|>0.1 \dot{\gamma}_{\max }\right)$

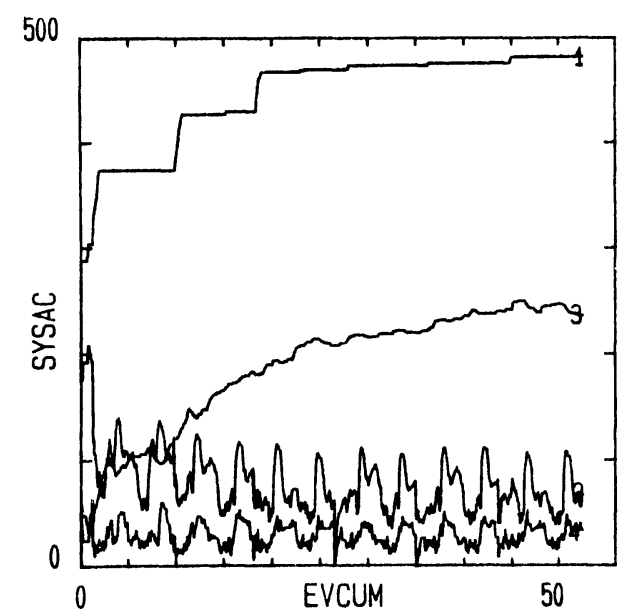

Figure 16 - Evolution du nombre de systèmes actifs et plastifiés au cours du chargement hors phase de la fig. 14 (Conventions de la fig.15).

Fig. 16 - Evolution of the number of active systems during out of phase loading (fig.14). curve $1: \mathrm{v}>0$; curve $2: \dot{\gamma}_{\mathrm{v}} \neq 0$.

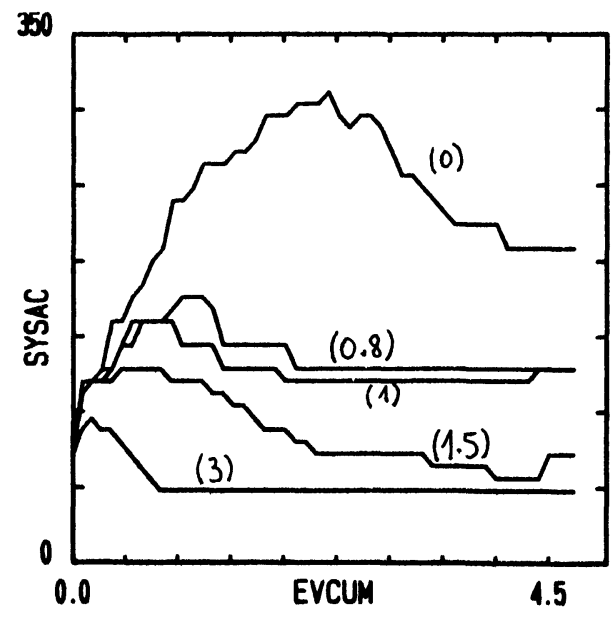

Figure 17 - Evolution du nombre de systèmes significativement actifs pour différentes valeurs des termes non diagonaux de $H$. (valeur du terme).

Fig. 17 - Evolution of the number of significantly active systems for different values of $H$ non diagonal terms. (Term's value).

\section{Conclusions}

On a montré une approche de type autocohérent suffisamment simplifiée pour être facilement manipulable dans les simulations numériques. Sa formulation en viscoplasticité lui permet de représenter le comportement plastique indépendant du temps (avec des valeurs extrèmes des coefficients) en même temps que le fluage ou la relaxation. Le temps calcul obtenu est raisonnbale pour un élément de volume isotrope contenant 48 grains, et permet d'envisager à moyen terme une application en calcul de structures.

La démarche employée pour exprimer le modèle qui consiste à utiliser au niveau des systèmes de glissement des lois de comportement macroscopiques classiques, peut être également utilisée pour les alliages à gros grains ou les monocristaux, que $l^{\prime}$ on retrouverait comme cas particuliers du modèle présenté. Dans le cas du monocristal en particulier, on sera dispensé des étapes 4 et 8 : on passe directement de la contrainte macroscopique aux cissions et on dispose donc d'un écrouissage "intragranulaire" sur chacun des systèmes de glissement, du type des modèles macroscopiques présentés en partie I. Sous sa forme actuelle, le modèle permet, avec seulement huit coefficients dépendants du matériau, de rendre compte des principaux phénomènes observés en plasticité cyclique. C'est ainsi qu'à côté de l'effet Bauschinger classique, on représente correctement le durcissement supplémentaire en chargement non proportionnel, l'effet de mémoire du trajet de déformation plastique et les distorsions des surfaces de charge, en montrant d'ailleurs que ces phénomènes sont liés.

Les améliorations à apporter portent essentiellement sur la relation qui définit la contrainte microscopique dans chaque grain (forme intégrée, "module" A constant), et sur la prise en compte des phénomènes de déconsolidation. 


\section{Notations}

Urdre des tenseurs: $\bar{T}$, ondre $1 ; \tilde{\mathrm{T}}$, ordre $2: \tilde{\tilde{T}}$, ondre 4 .

: designe le produit tensoriel contracte sur deux indices.

$\tilde{\Sigma}, \tilde{\Sigma}^{\prime}$ contraintes macroscopıques, deviateur associe

$\tilde{E}, \tilde{E}_{\mathrm{p}}, \tilde{\mathrm{E}}_{\mathrm{v}}$, deformations totale, plastıque, viscoplastıque

$\tilde{\mathbf{X}}, \tilde{\mathrm{X}}^{\prime}$, centre du domaine elastıque macroscopıque, deviateur associe

$J$, nvariant de Mises:J $(\tilde{\Sigma}-\tilde{X})=\left(1.5\left(. \tilde{\Sigma}^{\cdot}-\tilde{X}^{\prime}\right):\left(\tilde{\Sigma}^{\prime}-\tilde{X}^{\prime}\right)\right)^{0.8}$

$R$, rayon du domaine elastique macroscopique

$\tilde{n}$, normale aux equipotentielles viscoplastıques macroscopiques $\tilde{\mathbf{n}}=1 . \overline{5}\left(\tilde{\Sigma}^{1}-\tilde{\mathbf{X}}^{\cdot}\right) / \mathrm{J}$

$V$, deformation v1scoplastique macroscopique cumulée, $\dot{V}=\left(2 \dot{\tilde{E}}_{v}: \dot{\tilde{E}}_{v} / 3\right)^{0.8}$

$\tilde{\sigma}$, contraintes dans le grain $\&$

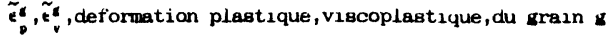

$\tau^{*}$, c18ajllement resolu sur le systeme 8 (plan $\vec{n}^{\circ}$, direction $\vec{i}^{\circ}$ $\tau^{*}=\tilde{m}^{*}: \tilde{\sigma}^{\alpha}$, avec $m_{1}=0.5\left(n_{1}^{*} \cdot 1_{j}^{*}+n_{j}^{*} \cdot 1_{1}^{*}\right)$

$x^{*}, r^{*}$, variables d'ecrourssage cinematique, 180 trope, sur le systeme

$v^{N}$, c1saillement viscoplastique cumule sur le systeme $s$, definı par : $\dot{v}=\left|\dot{r}_{v}\right|$

$\bar{r}, \bar{v}$, designent les vecteurs de composantes $r^{\circ}, v^{*}, a$ l'jnterieur d'un grain.

\section{Références}

|1 Conference on Conslitutive laws for Engineering Materials : Theory and Application, D.S. Desai, E. Krempl, P.D. Kiousis et T. Kundy éditeurs, Tucson, 5-8 janv. 87.

|2| Conference on Computational Plasticity : Models, Software and Applications. Barcelona, 6-10 avril 87.

|3| J. I.EMAitre, J.L. CHABOCHE, Mécanique des Matériaux Solides, Dunod, Paris (1985).

|4| J.L. CHABOCHE et G. ROUSSELIER, "On plastic and viscoplastic constitutive equations", part I et. II. ASME J. of pressure Vessel and Technology, vol. 105, p.153 (1983).

$|5|$ P.J. ARMSTRONG, C.o. FREDERICK, "A mathematical representation of the multiaxial Bauschinger effect", CEGB Report Report RD/B/N 731 (1966).

$|6| \mathrm{z}$. MROZ, "An Attempt to Describe the Behavior of Metals under Cyclic Loads Using a More General Workhardening Model", Acta Mech., 7, p.199 (1969).

$|7|$ o. WATANABE, S.N. ATLURI, "Internal tine, general internal variable and multi-yield- theories of plasticity and Creep : a unification of concepts", Int. J. of Plasticity, vol.2, p.37 (1986).

$|8|$ H. BURlet, G. CaIlletaud, "Modeling of cyclic plasticity in finite element codes", Conf. on Constitutive Laws for Engineering Materials : Theory and Application, D.S. Desal, E. Krempl, P.D. Kiousis, T. Kundu éditeurs, Tucson, (1987).

$|9|$ A. BALTOV, A. SAWCZUK, "A rule of anisotropic hardening", Acta Met. 1, p.81 (1965).

10| M. ROUSSET, "Surface seuil de plasticité : détermination automatique et modélisation", thèse Paris VI, (1985).

|11| P.G. HODGE, "General Theory of piecewise linear plasticity based on maximum shear". J. Mech. Phys. Solids, vol.5, p.242 (1957).

$|12| \mathrm{J}$. MANDEL, "Généralisation de la théorie de la plasticité de W.T. Koiter", Int. J. Sollds Structures, vol.1, p.273 (1965).

|13| W.T. KOITER, "Stress-Strain relations, uniqueness and variational theorems for elasto-plastic materials with a singular yield surface". Quarterly of Applied Mathematics, vol.11, $n^{\circ} 3, p .350$ (1953).

$|14|$ R. HILl, "Continuum Micro-Mechnisms of Elastoplastic Polycristals", J. Mech. Phys. Solids, vol.13, p.89 (1965).

|15| J.W. HUTCHINSON, "Elastic-Plastic Behaviour of Polycrystalline Metals and Composites". Proc. Royal Soc. Série A, vol.319, p.247 (1970).

$|16|$ M. BERVEiller, A. ZAOUI. "An Extension of the Self-Consistent Scheme to Plastically Flowing Polycrystals", J. Mech. Phys. Solids, vol.6, p.326 (1979).

$|17|$ F. KRONER, "Berechnung der Elastischen Konstanten des Vielkristalls aus den Konstanten des Einskristalls, " $z$. Physik, vol.151, p.504 (1958).

$|18|$ A. HIHI, M. BERVEILLER, A. ZAOUI, "Une nouvelle formulation de la modélisation auto-cohérente de la plasticité des polycristaux métalliques", Rapport GRECO Grandes Déformations et Endommagement $n^{\circ} 125(1984)$.

|19| G.M. BROWN, "A Self-Consistent Polycrystalline Model for Greep Under Combined Stress States", J. of Mech. and Phys, of Solids, vol.18, p.367 (1970).

20| J.w. HUTCHINSON, "Bounds and Self-Consistent Estimates for creep of Polycrystalline Materials", Proc. of the Royal Society, London, séries A, vol.348, p.10i (1976)

|21| G.J. WENG, "Self Consistent Determination of Time-Dependent Behavior of Metals", J. of Applied Mechanics, vol.48, p.41 (1981).

|22| L.M. BROWN, "Precipitation and Dispersion hardening", in proc. of 5 th. Int. Conf. on Strength of Metals and Alloys, vol.3, P. Haasen et al éditeurs, p.1551 (1980)

|23| J.W. MARTIN, "Micromechanisms in particle- hardened alloys", in Cambridge Solid State Science Series, R.W. Cahn, M.W. Thompson et Cambridge Solid State Science Series, R.W. Cahn, M.W.
I.M. Ward éditeurs, Cambridge University Press (1980).

$|24| \mathrm{H}$. MUGIRABI, "Cyclic deformation and fatigue of multi-phase materials", proc. of ath. Riso Int. Symp. on Melallurgy and Materials Science, p.65 (1983).

|25| R.E. STOLZ, R.M. PELLOUX, "The Bauschinger Effect in Precipitation Strengthened Aluminium Alloys". Metallurgical Transactions,

|26| R.J. ASARO, "Elastic-Plastic Memory and Kinematic-Type Hardening", Acta Metallurgica, vol.23, p.1255 (1975).

$|27|$ R. TAIllaRd, A. PINEAU, "Room Temperature Tensile Properties of Fe-19wt\% or Alloys Precipitation Hardened by the Intermetallic Compound NiAl", Materials Science and Engineering, 56, p.219 (1982).

|28| H.J. BUNGE, "Mathematische Methoden der Texturanalyse" Ak. Verlag, Berlin (1969).

|29| G. CAILLETAUD, H. KACZMAREK, H. POLICELLA, "Some elements on multiaxial behavior of 316 stainless steel at room temperature", Mechanics of Materials, vol.3, n⿳4, p.333 (1984).

|30| II.S. LAMBA, O.M. SIDEBOTTOM, "C.yclic plasticity for nonproportional paths", J. of Eng. Mat, and Tech, vol.100, p.96 (1978).

|31| A. BEnAllal, G. CAIlletaud, J.l. CHABOCHE, D. MARQUis, D. NOUAILHAS, M. ROUSSET, "Description and Modelling of Nonproportional effects in cyclic plasticity". Multiaxial Fatigue Conf., Sherfield (1985).

|32| J.L. CHABOCHE, K. DANG VAN, G. CORDIER, "Modelization of the strain memory effect on the cyclic hardening of 316 stainless stee1", SMIRT5, Berlin (1979).

|33| H.D. BUI, "Etude de l'évolution de la frontière du domaine élastique avec l'écrouissage et relations de comportement élasto-plastique des matériaux cubiques". Thèse, Univ. de Paris VI, (1969).

$|34|$ H. MUGHRABI, M. BAYERLEIN, H.J. CHIIST, "Microstructural foundation of cyclic stress-strain response and Masing behaviour", tion of cyclic stress-strain response and Masing behaviour",
Proc. 8th. Riso Int. Symp. "Constitutive Equations and their physical basis", Roskilde, Denmark (1987). 